\title{
Regional gray matter volume and structural network strength in somatic
} vs. non-somatic delusional disorders.

Huber, Markus; Wolf, Robert Christian; Lepping, Peter; Kirchler, Erwin; Karner, Martin; Sambataro, Fabio; Herrnberger, Bärbel; Corlett, Philip R.; Freudenmann, Roland W.

\section{Progress in Neuro-Psychopharmacology and Biological Psychiatry}

DOI:

10.1016/j.pnpbp.2017.11.022

Published: 01/03/2018

Peer reviewed version

Cyswllt i'r cyhoeddiad / Link to publication

Dyfyniad o'r fersiwn a gyhoeddwyd / Citation for published version (APA):

Huber, M., Wolf, R. C., Lepping, P., Kirchler, E., Karner, M., Sambataro, F., Herrnberger, B., Corlett, P. R., \& Freudenmann, R. W. (2018). Regional gray matter volume and structural network strength in somatic vs. non-somatic delusional disorders. Progress in NeuroPsychopharmacology and Biological Psychiatry, 82, 115-122.

https://doi.org/10.1016/j.pnpbp.2017.11.022

\footnotetext{
Hawliau Cyffredinol / General rights

Copyright and moral rights for the publications made accessible in the public portal are retained by the authors and/or other copyright owners and it is a condition of accessing publications that users recognise and abide by the legal requirements associated with these rights.

- Users may download and print one copy of any publication from the public portal for the purpose of private study or research.

- You may not further distribute the material or use it for any profit-making activity or commercial gain

- You may freely distribute the URL identifying the publication in the public portal ?
}

Take down policy

If you believe that this document breaches copyright please contact us providing details, and we will remove access to the work immediately and investigate your claim. 


\section{Regional gray matter volume and structural network strength in}

\section{somatic vs. non-somatic delusional disorders}

Markus Huber ${ }^{1 \#}$, Robert Christian Wolf ${ }^{2 \#^{*}}$, Peter Lepping ${ }^{3}$, Erwin Kirchler ${ }^{1}$, Martin Karner ${ }^{4}$, Fabio Sambataro ${ }^{5}$, Bärbel Herrnberger ${ }^{6}$, Philip R Corlett ${ }^{7}$, Roland W Freudenmann ${ }^{6}$

Betsi Cadwaladr University Health Board, Maelor Hospital, Centre for Mental Health and Society, Wrexham, Wales, UK

$4 \quad$ Department of Radiology, General Hospital Bruneck, South Tyrol, Italy

5 Department of Experimental \& Clinical Medical Sciences, Udine University, Italy

$6 \quad$ Department of Psychiatry and Psychotherapy III, Ulm University, Germany

7 Department of Psychiatry, Yale University, Connecticut Mental Health Center, New Haven, CT, USA

*Corresponding author: Robert Christian Wolf, MD

Center for Psychosocial Medicine

Department of General Psychiatry

Heidelberg University, Vosstrasse 4,

69115 Heidelberg, Germany

E-mail: christian.wolf@uni-heidelberg.de

Tel: +49-6221-568061, Fax: +49-6221-564481

\#these authors contributed equally to this manuscript

Short title: Abnormal brain structure in delusional disorders 


\section{Abstract}

Background: Monothematic delusional disorders are characterized by a single tenacious belief. They provide a great opportunity to study underlying brain structures in the absence of confounding symptoms that accompany delusions in schizophrenia. Delusional beliefs include persecution, jealousy or somatic delusions including infestation. It is unclear whether specific delusional content is associated with distinct neural substrates.

Methods: We used magnetic resonance imaging in patients presenting with somatic vs. nonsomatic delusional disorders. Patients with delusional infestation (DI, $n=18$ ), and individuals with non-somatic delusional disorders $(n=19)$ were included, together with healthy volunteers $(n=20)$. Uni- and multivariate techniques for structural data analysis were applied to provide a comprehensive characterization of abnormal brain volume at both the regional and neural network level.

Results: Patients with DI showed lower gray matter volume in thalamic, striatal (putamen), insular and medial prefrontal brain regions in contrast non-somatic delusional disorders and healthy controls. Importantly, these differences were consistently detected at regional and structural network level. Compared to healthy controls, patients with delusional disorders other than DI showed lower gray matter volume in temporal cortical regions.

Conclusion: The data support the notion of dysfunctional somatosensory and peripersonal networks that could mediate somatic delusions in patients with DI in contrast to delusional disorders without somatic content. The data also suggest putative content-specific neural signatures in delusional disorders and in delusion formation per se.

Key words: delusional disorders; somatic delusions; MRI, voxel-based morphometry; source-based morphometry; peripersonal space 


\section{Introduction}

Delusional disorders are psychotic disorders characterized by fixed beliefs as a single symptom, sometimes accompanied by hallucinations related to the delusional theme. Therefore, they can be considered as model diseases for single-symptom-based neuroscientific research in psychotic disorders (Corlett et al., 2010). However, delusional disorders are little investigated in neuroscience because patients with these disorders usually fail to consent or adhere to any study protocol (Ahmed and Bewley, 2013; Skelton et al., 2015). Tenacious beliefs in delusional disorders are related to persecution, jealousy, grandiosity, love, nihilism, and misidentification of self and others as well as somatic delusions (delusional disorder somatic type).

A prominent form of delusional disorder somatic type is delusional infestation (DI), also known as delusional parasitosis or Ekbom syndrome (Freudenmann and Lepping, 2009). Patients with DI have the fixed belief that small living or (much rarer) inanimate pathogens such as insects or worms infest their body. They also "feel" them crawling, stinging, biting on the skin or elsewhere in the body, although there is no medical evidence of their presence (Freudenmann and Lepping, 2009; Hylwa et al., 2011). The clinical features of DI often show an overlap between abnormal beliefs (somatic delusions) and abnormal perceptions (Baker et al., 1995). This has been highlighted in the DSM-5 diagnostic criteria for delusional disorder (DSM-5: 297.1), where symptoms of DI are used to illustrate characteristic presence of delusions and hallucinations that mirror these delusions in this group of psychotic disorders ("the sensation of being infested with insects associated with delusions of infestation"). Formerly, DI has been seen rather a disorder of perception ("chronic tactile hallucinosis" according to Bers and Conrad, 1954) or an "organic hallucinosis" (ICD-10). Today, it is mostly referred to as a disorder of thought and reasoning (Trabert, 1995; Freudenmann and Lepping, 2009; Lepping et al., 2015). DI can occur as a primary monothematic delusional disorder (primary DI) or, more commonly, as a secondary delusion. In secondary DI the delusion arises in the context of another major medical, neurological or 
psychiatric disorder that affects brain functioning or it is associated with the use of illicit or prescribed substances, usually with dopaminergic mechanism of action (Huber et al., 2007, Dunn et al., 2007; Beach et al., 2014).

A number of case reports suggested frontal, temporo-parietal, striatal and thalamic brain dysfunction in DI let us propose a hypothetical disease model in 2009 (Huber et al., 2008; Freudenmann and Lepping, 2009). We recently introduced the first structural magnetic resonance imaging (MRI) reports using univariate (i.e. voxel-based morphometry [VBM], and multivariate, i.e. source-based morphometry [SBM]) statistical methods for structural data analyses (Wolf et al., 2013; Wolf et al., 2014). Our findings supported the hypothesis that the delusional belief to be infested with pathogens is likely associated with disrupted prefrontal control over somato-sensory representations.

Here, we report the first transnosologic MRI study investigating gray matter volume (GMV) abnormalities in patients with DI and individuals presenting with non-somatic delusions compared to healthy controls. We specifically aimed at identifying structural brain abnormalities that differ within the broad category of delusional disorders when specified by the delusional content, i.e. somatic vs. non-somatic. Whole-brain univariate VBM and multivariate statistical techniques were employed, complemented by a region-of-interest (ROI) approach, to provide a comprehensive characterization of abnormal brain volume at both the regional and neural network level. We predicted that in contrast to patients with nonsomatic delusions and healthy controls, patients with DI will show more pronounced structural deficits in subcortical (dorsal striatum, thalamus) and frontal brain regions reflecting aberrant neuroanatomical pathways underlying somatic delusions, especially within the context of DI. 


\section{Methods}

\subsection{Participants}

We investigated 38 patients presenting with a delusional disorder according to DSM-5 criteria. All participants were right-handed, as identified by their dominant writing hand. Participants were recruited at the Psychiatric Department of the General Hospital Bruneck/South Tyrol, Italy and contacted by phone with the help of the local electronic hospital information system.

We included 18 patients with a delusional disorder, somatic type (DI-group) and 20 patients with non-somatic delusional disorders (NonDI-group). One NonDI-participant was discarded due to insufficient MRI data quality (see section 2.3 below). The healthy control (HC) group included 20 healthy volunteers. 16 DI-patients and $16 \mathrm{HC}$ were already considered in previous works (Wolf et al., 2013 and 2014). None of the NonDI-patients were considered in previous studies.

The DI-group consisted of 10 females and 8 males with a mean age of 74.3 (standard deviation, $\mathrm{SD}=8.9$ ) years. In the DI-group 6 patients was classified as primary $\mathrm{DI} ; 3$ cases were associated with other mental disorders (2x major depression, $1 \mathrm{x}$ cyclothymia); and 9 cases were classified as secondary to various medical conditions (5x subcortical vascular encephalopathy, 1x severe hearing loss/blindness, 1x hyperthyroidism, 1x Parkinson's disease, $1 \mathrm{x}$ iron deficiency). Mean disease duration was 6.6 years $(S D=8.9)$. All patients received antipsychotic treatment (mean chlorpromazine [CPZ] equivalents $=258.4, \mathrm{SD}=$ 125.0, Woods, 2003).

The NonDI-group that was considered for further analyses included 14 females and 5 males with a mean age of $56.1(S D=14.9)$ years. NonDI-patients presented with the following nonsomatic delusional content: persecution only $(n=9)$, mixed persecution/poisoning $(n=4)$, jealousy ( $n=2$, without any association to any substance-use disorder), poverty $(n=2)$, hypochondria $(n=2)$. None of the patients fulfilled diagnostic criteria for an underlying affective disorder. Mean disease duration was 13.3 years $(S D=11.2)$. Three patients were 
unmedicated. Sixteen patients received antipsychotic treatment (mean chlorpromazine [CPZ] equivalents $=171.3, \mathrm{SD}=96.4)$.

$\mathrm{HC}$ included 12 females and 8 males with a mean of $70.4(\mathrm{SD}=11.9)$ years. $\mathrm{HC}$ were considered if they had no psychiatric or neurological history or severe medical condition. None of the $\mathrm{HC}$ had a history of psychotropic drug treatment.

None of the participants had a history of substance-use disorder or met criteria for major neurocognitive disorder. All patients were under treatment with antipsychotics for at least one year prior to the MRI recording.

This study was carried out in accordance with The Code of Ethics of the World Medical Association (Declaration of Helsinki) for experiments involving humans. All participants gave written informed consent as approved by the local institutional authority (Health District Bruneck/South Tyrol-Italy).

\subsection{Structural neuroimaging and data acquisition}

Structural data were acquired in the Department of Radiology at the General Hospital Bruneck, South Tyrol, Italy, using an MRI system at 1.0 Tesla (Philips INTERA, Release 11, Best, The Netherlands). The MRI parameters of the 3D T1 gradient echo recalled (fast field echo, FFE) sequence were as follows: TE = $6.9 \mathrm{~ms} ; \mathrm{TR}=25 \mathrm{~ms} ; \mathrm{FOV}=230 \mathrm{~mm}$ [AP], 172 $\mathrm{mm}[\mathrm{RL}]$; resolution $=0.9 \mathrm{~mm}^{3} ;$ number of slices $=170$.

\subsection{Data pre-processing}

A toolbox for VBM (http://dbm.neuro.uni-jena.de/vbm8/) running within the Statistical Parametric Mapping software package version 8 (SPM8; http://www.fil.ion.ucl.ac.uk/spm) was used (Ashburner and Friston, 2000; Xu et al., 2009; Kasparek et al., 2010). Data quality procedures included visual inspection and checking for sample homogeneity which led to the exclusion of one NonDI-participant from subsequent analyses (details on data preprocessing are provided as supplementary material). In brief, each participant's original T1 
image was segmented into gray matter (GM) and white matter as well as cerebrospinal fluid. Modulated normalized GM segments were smoothed using an $8 \mathrm{~mm}$ Full Width at Half Maximum (FWHM) Gaussian kernel prior to between-group analyses at the $2^{\text {nd }}$ level.

\subsection{VBM whole-brain analysis}

A full factorial model was used including group as a factor; treating age and gender as nuisance variables. An absolute threshold of 0.1 was used to prevent effects occurring at tissue border regions. A main analysis was conducted including HC, DI-patients and the entire NonDI-patient cohort. To account for potential phenomenological diversity in the NonDI-patient sample a second analysis was performed including HC, DI-patients and a subsample of NonDI-patients, i.e. those presenting with prominent paranoid (persecutory) delusions only $(n=13)$. The effect of group was assessed using an F-contrast $(p<0.001$ uncorrected at the voxel level, $p<0.05$ corrected for spatial extent). Post-hoc t-tests were used to assess differences between the groups at $p<0.001$ (uncorrected at the voxel level, $p<0.05$ corrected for spatial extent). Anatomical regions emerging from the $2^{\text {nd }}$ level analyses were labelled according Talairach Daemon (TD) labels and the Automatic Anatomical Labeling Atlas (AAL) (Tzourio-Mazoyer et al., 2002). The stereotaxic coordinates reported in Table 1 were derived from all anatomically distinct regions within in a given cluster surviving the chosen significance threshold. All coordinates are reported in Montreal Neurological Institute (MNI) space. To facilitate comparisons with recent neuroimaging research in nonsomatic delusional disorders (Vicens et al., 2016), whole-brain $2^{\text {nd }}$-level analyses were recalculated using unmodulated segments. For this purpose, we repeated the VBM segmentation to obtain unmodulated normalized data. Using these data, again, a full factorial model was employed where age, gender and total intracranial volume were used as nuisance variables. A significance threshold of $p<0.001$ (uncorrected at the voxel level, $p<0.05$ corrected for spatial extent) was used.

\subsection{ROI analysis}


In addition to whole-brain data we were also interested in region-wise comparisons between the groups. Since we were primarily interested in the neurobiology underlying DI, we performed ROI-based analyses using a distinct set of subcortical and cortical regions whose contributions to tactile/sensory processing and control is well-known. For this purpose we first entered all GMV images into a one sample t-test model adjusted for age and gender and restricted these analyses to the following set of brain areas, as defined by the AAL atlas: thalamus, bilateral putamen, insula, post-central cortex, middle frontal cortex and anterior cingulate cortex. These ROls were chosen based on the neurobiological model proposed by us in 2009 and subsequent studies (Freudenmann and Lepping, 2009; Wolf et al., 2013). Subsequently we extracted cluster-wise mean GMV parameter estimates representing the extent of regional volume for each individual. These analyses were performed offline using the Statistical software package (Version 10) using an ANOVA model. A nominal $p<0.05$ was defined, FDR-corrected for multiple comparisons (Benjamini et al., 2001). Fisher's LSD tests were used post-hoc $(p<0.05)$.

\subsection{Source-based morphometry}

We used SBM to investigate the strength of structural networks in between the patient groups as well as between patients and controls (detailed information on SBM is presented as supplementary material). Using individual GMV segments (see section 2.3) a spatial independent component analysis (ICA) was computed. We used the SBM algorithm as implemented in the "Group ICA for f-MRI Toolbox" [GIFT; http://mialab.mrn.org/software/gift] (Xu et al. 2009). Nine independent components were identified, as estimated by minimal description length (MDL) criteria (Li et al., 2007). To increase the stability of the estimated components, we used the Icasso algorithm and repeated the ICA estimation 50 times with bootstrapping and permutation (Himberg et al., 2004). Using ICA, each GMV image was converted into a one-dimensional vector which was arrayed into one 57-row subject/bysegment data matrix. This matrix was decomposed into one mixing and one source matrix. Between-group comparisons were performed using mixing matrix indices. Component 
selection and post-hoc between-group comparisons were based on ANOVA models on every column of the mixing matrices. The ANOVA models included a group factor representing HC, DI-patients and NonDI-patients. Analyses were performed offline using the Statistical software package (Version 10). A nominal $p<0.05$, was defined, FDR-corrected for multiple comparisons. Fisher's LSD tests were used post-hoc $(p<0.05)$. For component visualization the source matrix was reshaped back to a three-dimensional image, scaled to unit standard deviations ( $Z$ maps) and threshold at $Z>2.5$. Maps of interest were overlaid onto a MNI normalized anatomical template. Anatomical denominations and stereotaxic coordinates were obtained from clusters above a threshold of $Z=2.5$ by linking the SBM output to the TD data base (www.talairach.org/daemon.html).

\section{Results and statistical analyses}

\subsection{Demographic data}

The patient groups did not significantly differ from each other with respect to gender $(p=0.10)$, but DI-patients were older than NonDI-patients $(p=0.000) . H C$ and DI-patients did not significantly differ in age $(p=0.23)$ and gender $(p=0.56)$. NonDI-patients were significantly younger than $\mathrm{HC}(\mathrm{p}=0.002)$ but they did not significantly differ from $\mathrm{HC}$ with respect to gender $(p=0.23)$. Disease duration in DI-patients was shorter compared to the NonDI-group $(p=0.026)$. The patient groups differed in terms of CPZ equivalents $(p=$ 0.014), with lower mean CPZ equivalents in the DI group compared to NonDI.

\subsection{Whole brain VBM analysis}

A group effect was found in bilateral parahippocampal, lateral temporal and fusiform cortices, left insula and anterior cingulate cortex (see Figure 1-supplement and Table 1-supplement for detailed stereotaxic coordinates and Z-scores). 
These effects were driven by lower GMV in DI-patients compared to HC: in post-hoc comparisons, lower GMV in patients with DI was found in medial and lateral prefrontal areas, medial and lateral regions of the temporal lobe (including the hippocampus and parahippocampus), bilateral fusiform cortices, subcallosal and anterior cingulate cortices, bilateral insula, left putamen, left thalamus and left cerebellum (see Figure 1 and Table 1 for detailed stereotaxic coordinates and Z-scores). In DI-patients, there were no regions showing significantly higher GMV compared to HC.

Compared to HC, patients with delusions other than DI (NonDI-group) showed lower GMV in the left inferior temporal cortex $(x=-42, y=-1, z=-39, Z=4.98, k=1328)$ and the right fusiform cortex $(x=26, y=-69, z=-8, Z=4.98, k=417$ ) (see Figure 1). In these patients, there were no regions showing significantly higher GMV compared to HC. No significant differences emerged when DI-patients were contrasted to NonDI-patients. When analyses were repeated using a subgroup of patients with prominent paranoid (persecutory) delusions only, a similar pattern of GMV volume loss was obtained. Findings in the DI-patient group compared to $\mathrm{HC}$ remained largely unchanged. In the NonDI-patients subgroup with prominent paranoid (persecutory) delusions lower GMV compared to $\mathrm{HC}$ was found in the left inferior temporal cortex $(x=-42, y=-1, z=-39, z=4.33, k=481)$ (see Figure 1). None of the patient groups showed higher GMV compared to HC. The patient groups did not significantly differ from each other.

Using unmodulated segments, findings in the DI-group vs. HC and vs. NonDI-patients remained largely unchanged $(p<0.001$, uncorrected). Compared to $\mathrm{HC}$, NonDI-patients showed lower GMV in the left insula $(x=-45, y=-13, z=-7, Z=4.13, k=481)$, the left inferior temporal cortex $(x=-41, y=0, z=-39, z=3.85, k=260)$, the right fusiform cortex $(x=23, y=-42$, $\mathrm{Z}=-11, \mathrm{Z}=3.75, \mathrm{k}=292$ ), in a cluster comprising the right superior temporal gyrus and the parahippocampal gyrus ( $x=33, y=18, z=-33, Z=3.72$ and $x=33, y=11, z=-32, Z=3.60, k=421$ ) and in the right inferior temporal gyrus $(x=54, y=-35, z=-27, Z=3.70, k=273$ ) (see Figure 3supplement). 


\subsection{ROI analysis}

Except for the bilateral middle frontal cortex a significant group effect was found for each of the remaining ROIs. Post-hoc tests revealed that patients with DI had significantly lower volumes compared to $\mathrm{HC}$ and patients with other delusions (NonDI-group) in the bilateral putamen, thalamus, insula and the anterior cingulate cortex. For the bilateral postcentral cortex post-hoc tests revealed significantly lower GMV in patients with DI compared to NonDI-patients. In all post-hoc tests, there were no significant differences between $\mathrm{HC}$ and NonDI-patients (detailed statistics and GMV plots showing regional extent of GMV across the groups are available on request).

\subsection{Network SBM analysis}

Nine components were estimated using MDL criteria. Three of these components showed a significant effect of group ( $p<0.05$, FDR-corrected). Two components were chosen for further post-hoc analyses (see Figure 2 and Table 2 for detailed anatomical labels, coordinates and Z-scores); one component clearly signalled head movement artefacts and was thus discarded (see Figure 2-supplement). The first significant $(F(2,54)=3,7671, p=.0294)$ component of interest (COI1, "striatal network") displayed a pattern of predominantly bilateral striatal and thalamic regions together with areas of the frontal cortex. Post-hoc tests revealed that patients with DI had lower GMV than HC $(p=0.013)$ and patients with other delusions (NonDI-group) $(\mathrm{p}=0.036)$. There were no differences between $\mathrm{HC}$ and the NonDI-group $(p=0.689)$. The second significant $(F(2,54)=5,3625, p=.0075)$ component of interest $(\mathrm{COI} 2$, "frontotemporal network") included predominantly the bilateral insula, medial and lateral temporal areas and anterior cortical midline regions. Post-hoc tests revealed that patients with DI had lower GMV than $\mathrm{HC}(\mathrm{p}=0.026)$ and patients with delusions other than DI $(p=0.002)$. There were no differences between $\mathrm{HC}$ and patients from the NonDI-group $(p=0.348)$. 


\section{Discussion}

This structural MRI study investigated brain structure of patients with somatic and nonsomatic delusional disorders at the regional level and at the level of structural network expression HC. Our results provide first evidence that patients with somatic delusions, i.e. DI, differ from patients with non-somatic delusional disorders and HC. More specific, DI patients exhibited a pattern of lower GMV in fronto-thalamo-striatal and frontotemporal regions. In contrast to our previous studies, our findings indicate that the brain changes observed in DI are more pronounced in this form of delusional disorder, in contrast to delusions with other monothematic content. Importantly, the volume differences found in the patient groups were not restricted to the regional level, as shown by whole-brain VBM and pre-defined ROI analyses, the latter providing clear differences between individuals with and without somatic delusions. At the level of structural network expression (SBM) two networks were identified as abnormal in patients with DI in contrast to the patients with other delusional disorders and HC. In both components of interest, striatal and frontotemporal, patients with DI showed significant lower GMV compared to both patients with non-somatic delusions and HC.

The findings share some key features with those seen in the unfortunately small number of studies imaging patients with delusional disorders in general (Su et al., 2001; Kunert et al., 2007; Vicens et al., 2016). So far, brain correlates of delusions are studied systematically mainly in patients with schizophrenia (Knobel et al., 2008). Interestingly, imaging studies in somatic delusions in patients with schizophrenia found GMV-abnormalities (frontal, insular, thalamus) similar to the present study in DI (Cascella et al., 2001; Spalletta et al., 2013). Patients with non-psychotic chronic skin manipulations, behaviours typically seen in DI (itchscratch-cycle), also showed brain abnormalities in thalamo-striatal and orbitofrontal regions (Schneider et al., 2008; Mochizuki et al., 2009). This may suggest that common neural pathways could underlie the expression of somatosensory phenomena, probably regardless of nosological considerations. 
We provide further evidence for pathology in the dorsal, somatic striatum (putamen) in DI. Importantly, the abnormal striatal volume in the DI-patients was confirmed (in contrast to the NonDI-patients and $\mathrm{HC}$ ) by all our performed distinct analyses (whole-brain VBM, ROIanalysis and SBM), suggesting a robust finding in DI. This supports the notion that the basal ganglia, particularly the dorsal striatum (putamen), are involved in various perception processes such as in visuo-tactile perception and sensory predictions (Ladavas et al., 1998; Yoo et al., 2003; Colder, 2015). The putamen is known as an essential node of the so called "Peripersonal Space Network" relevant for the neural integration of visual with tactile information (visuo-tactile perception) near, but outside the body (Graziano and Gross, 1993, Gentile et al., 2011; Brozzoli et al., 2014). This network is well researched with neuroimaging in human beings and includes frontal, parietal, insular and subcortical (putamen) brain areas where visual and tactile signals converge (Brozzoli et al., 2012; Di Pellegrino and Ladavas, 2015). The so-called "Peripersonal Space Network" is thought to play a fundamental role in the representation of the space in the immediate vicinity of the body $(10-20 \mathrm{~cm})$, permitting the construction of a special cerebral encoding of the space that lies in the boundary zone between objects near the body and the body itself (Di Pellegrino and Ladavas, 2015; de Vignemont and lannetti, 2015). Thus, DI may underlie a specific malfunction of the so-called "Peripersonal Space Network".

In the present DI sample, the bilateral postcentral gyrus showed lower GMV in the ROIbased analyses only. This was unexpected given that DI patients usually suffer from intense sensations in their skin or body. However, converging evidence suggests that perception of touch in humans does not only require the postcentral gyrus and parietal operculum but also involves the insular cortex and putamen together with prefrontal structures as well as superior temporal and limbic structures (Nagy et al., 2006; Auksztulewicz et al., 2012 Preusser et al., 2015). We also found evidence for structural brain changes in insular cortex, a brain region known to mediate feelings of disgust and as a potential source of interoceptive predictions (Wicker et al., 2003; Craig, 2003; Paulus and Stein, 2009). Similarly, a recent functional MRI study in DI patients found changes in neural activity not only in frontal but also 
insular and temporal cortices next to limbic structures, such as the amygdala (Eccles et al,. 2015). Structural brain changes in these regions in people with delusions would be consistent with disturbed interaction between top-down expectation and bottom-up input that may cause prediction errors and delusional beliefs (Schmack et al., 2013; Barrett and Simons, 2015; Pia et al., 2015). However, disturbances in the primary somatosensory cortex and right frontal cortex would be more consistent with the two-factor theory of monothematic delusions (Davies et al., 2001; Coltheart, 2010).

The frontostriatal abnormalities support the notion that somatic delusions could essentially originate prefrontal failure to optimize visuo-tactile uncertainty, leading to an impaired judgment with abnormal delusional beliefs and errors of probabilistic reasoning. In this model, dysfunctional processing in the dorsal striato-subcortical loop could explain abnormal visuo-tactile perceptions (hallucinations) presumably mediated by the damaged putamen (dorsal "somatic" striatum). This may support that delusions originate from a neural network failure between top-down and bottom-up processing as mismatches between expectations and experience (aberrant prediction errors) (Fletcher and Firth, 2009; Ding and Gold, 2013; Colder, 2015; Adams et al., 2016; Chanes and Barrett, 2016). Yet it is very challenging to distinguish two-factor and predictive coding explanations of delusions on the basis of structural data alone, as they make different predictions about information processing rather than brain structure (Corlett et al., 2007 and 2010; Corlett 2015).

Potential limitations of this study include the modest sample sizes and the use of psychotropic medication in the patient samples. Nevertheless, given the difficulties to motivate patients with monothematic delusions (and especially those with $\mathrm{DI}$ ) to participate in a study, we consider the present sample sizes as relatively large compared with the few other currently available studies investigating the neural underpinnings of delusional disorders. Similarly, the presence of an antipsychotic agent in these patients reflects common clinical practice. We are not aware of any study so far presenting data from antipsychotic-naïve DI-patients, as much as we are not aware of studies which investigated antipsychotic-naïve patients with delusional disorders other than DI. Antipsychotic treatment 
can have an impact on brain structure. Yet the differences in GMV change and structural network strength, as observed in DI vs. nonDI, cannot be solely attributed to antipsychotic treatment, since CPZ equivalents differed between the patient groups, with DI-patients being treated with lower dosages of antipsychotics compared to NonDI. Age and disease duration need to be considered as potentially relevant variables as well, since the NonDI-group was significantly younger compared to both DI-patients and HC, and since NonDI-patients presented with longer disease duration. However, we included age as nuisance variable in all analyses, and it is difficult to assign more pronounced gray matter volume loss to shorter disease duration, as observed in the DI-group. Also, the DI group is etiologically more heterogeneous than the NonDI-group, as it includes patients with affective disorders and various medical conditions. Nevertheless, we have previously shown that at least in terms of structural network strength, frontostriatal and insular dysfunction in DI-patients is independent of etiological considerations Wolf et al., 2014). In the context of our study, we emphasize the clinical phenotype and its potential neural substrates and not a specific (putative) etiology, keeping in mind that such approaches could potentially hamper transnosological observations. Eventually, we also acknowledge that any hypotheses regarding functional mechanisms are speculative at this stage because we are inferring from structural data. Functional and structural abnormalities in patients with delusional disorders can co-occur (Vicens et al., 2016). It is, however, unclear whether structural deficits drive functional abnormalities or whether two distinct levels of neural dysfunction (i.e. structure and function) differentially contribute to symptom expression. These questions are clearly of outstanding interest, and future research in delusional disorders clearly has to consider these distinct levels of neuropathology and their interrelationships. 


\section{Conclusion}

Keeping this study's limitations in mind, we provide a comprehensive analysis of brain structure in patients with somatic and non-somatic delusional disorders. Our findings support the notion of dysfunctional sensorimotor and peripersonal neural pathways that could mediate somatic delusions in patients with $\mathrm{DI}$ in contrast to non-somatic delusional content. The data suggest putative content-specific neural signatures in delusional disorders and in delusion formation per se. Future multimodal neuroimaging studies, i.e. combined functional and structural MRI protocols, are desirable address this specific hypothesis, and to establish robust links between specific symptom expression, i.e. delusional content, and its neural underpinnings. 


\section{Acknowledgments}

We thank all our study participants for their understanding and willingness to participate in this study. We would also thank the General Hospital Bruneck, South-Tyrol, Italy, for the support and approval of this study.

\section{Funding}

This research did not receive any specific grant from funding agencies in the public, commercial, or not-for-profit sectors. 


\section{References}

Adams, R.A., Huys, Q.J., Roiser, J.P., 2016. Computational Psychiatry: towards a mathematically informed understanding of mental illness. J Neurol Neurosurg Psychiatry 87, 53-63.

Ahmed, A., Bewley, A., 2013. Delusional infestation and patient adherence to treatment: an observational study. Br J Dermatol 169, 607-610.

Ashburner, J., Friston, K.J., 2000. Voxel-based morphometry-the methods. Neuroimage 11, 805-821.

Auksztulewicz, R., Spitzer, B., Blankenburg, F., 2012. Recurrent neural processing and somatosensory awareness. J Neurosci 32, 799-805.

Baker, P.B., Cook, B.L., Winokur, G., 1995. Delusional Infestation: the interface of delusions and hallucinations. Psychiatr Clin North Am 18, 345-361.

Barrett, L.F., Simons, W.K., 2015. Interoceptive predictions in the brain. Nat Rev Neurosci 16, 419-20. Beach, S.R., Kroshinsky, D., Kontos, N., 2014. Case records of the Massachusetts General Hospital. Case 37-2014. A 35-year-old woman with suspected mite infestation. N Engl J Med 371, 2115-2123.

Benjamini, Y., Drai, D., Elmer, G., Kafkafi, N., Golani, I., 2001. Controlling the false discovery rate in behaviour genetics research. Behav Brain Res 125, 279-284.

Bers, N., Conrad, K., 1954. Die chronische taktile Halluzionose. Fortschr Neurol Psychiatr 22, 254270.

Brozzoli, C., Ehrsson, H.H., Farne, A., 2014. Multisensory representation of space near the hand: from perception to action and interindividual interactions. Neuroscientist 20, 122-135.

Brozzoli, C., Makin, T.R., Cardinali, L., Holmes, N.P., Farne, A., 2012. Peripersonal Space: A Multisensory Interface for Body-Object Interactions. In: Murray MM, Wallace MT, editors. The neural Bases of Multisensory Processes. Boca Raton (FL) CRP Press Taylor \& Francis. Frontiers in Neuroscience: Chapter 23.

Cascella, N.G., Gerner, G.J., Fieldstone, S.C., Sawa, A., Schretlen, D.J., 2001. The insula-claustrum region and delusions in schizophrenia. Schizophr Res 133, 77-81.

Chanes, L., Barrett, L.F., 2016. Redefining the Role of Limbic Areas in Cortical Processing. Trends Cogn Sci 20, 96-106. 
Colder, B., 2015. The basal ganglia select the expected sensory input used for predictive coding. Front Comput Neurosci 23:9, 119.

Coltheart. M., 2010. The neuropsychology of delusions. Ann NY Acad Sci 1191, 16-26.

Corlett, P.R., Murray, G.K., Honey, G.D., Aitken, M.R., Shanks, D.R., Robbins, T.W., Bullmore, E.T., Dickinson, A., Fletcher, P.C., 2007. Disruped prediction-error signal in psychosos: evidence for an associative account of delusions. Brain 130; 2387-2400.

Corlett, P.R., Taylor, J.R., Wang, XJ, Fletcher, P.C., Krystal, J.H., 2010. Toward a neurobiology of delusions. Prog Neurobiol 92, 345-369.

Corlett, P.R., 2015. Answering some phenomenal challenges to the prediction error model of delusions. World Psychiatry 14, 181-183.

Craig, A.D., 2003. Interoception: the sense of the physiological condition of the body. Curr Opin Neurobiol 13, 500-505.

Davies, M., Coltheart, M., Langdon, R., Breen, N., 2001. Monothematic delusions: Toward a two-factor account. Philosophy, Psychiatry \& Psychology 8, 133-158.

de Vignemont, F., lannetti, GD., 2015. How many peripersonal space? Neuropsychologia 70; 327-334. Di Pellegrino, G., Ladavas, E., 2015. Peripersonal space in the brain. Neuropsychologia 66, 126-133. Ding, L., Gold, J.I., 2013. The basal ganglia's contributions to perceptual decision making. Neuron 79; 640-649.

Dunn, J., Murphy, M.B., Fox, K.M., 2007. Diffuse pruritic lesions in a 37-year-old man after sleeping in an abandoned building. Am J Psychiatry 164, 1166-1172.

Eccles, J.A., Garfinkel, S.N., Harrison, N.A., Ward, J., Taylor, R.E., Bewley A.P., Critchley H.D., 2015. Sensations of skin infestation linked to abnormal frontolimbic brain reactivity and differences in selfrepresentations. Neuropsychologia 77, 90-96.

Fletcher, P.C., Frith, CD., 2009. Perceiving is believing: a Bayesian approch to explaining the positive symptoms of schizophrenia. Nat Rev Neurosci 10, 48-58.

Freudenmann, R.W., Lepping, P., 2009. Delusional Infestation. Clin Microbiol Rev 22, 690-732.

Gentile, G., Petkova, V.I., Ehrsson, H.H., 2011. Integration of visual and tactile signals from the hand in the human brain: an FMRI study. J Neurophysiol 105, 910-922. 
Graziano, M.S., Gross, C.G., 1993. A bimodal map of space: somatosensory receptive fields in the macaque putamen with corresponding visual receptive fields. Exp Brain Res 97, 96-109.

Himberg, J., Hyvärinen, A., Esposito, F., 2004. Validating the independent components of neuroimaging time series via clustering and visualization. Neuroimage 22, 1214-1222.

Huber, M., Karner, M., Kirchler, E., Lepping, P., Freudenmann, R.W., 2008. Striatal lesions in delusional parasitosis revealed by magnetic resonance imaging. Prog Neuropsychopharmacol Biol Psychiatry 32, 1967-1971.

Huber, M., Kirchler, E., Karner, M., Pycha, R., 2007. Delusional parasitosis and the dopamine transporter. A new insight of etiology? Med Hypotheses 68, 1351-1358.

Hylwa, S.A., Bury, J.E., Davis, M.D., Pittelkow, M., Bostwick, J.M., 2011. Delusional infestation, including delusions of parasitosis: results of histologic examination of skin biopsy and patient-provided skin specimens. Arch Dermatol 147, 1041-1045.

Kasparek, T., Marecek, R., Schwarz, D., Prikryl, R., Vanicek, J., Mickl, M., Ceskova, E., 2010. Sourcebased-morphometry of gray matter volume in men with first-episode schizophrenia. Hum Brain Mapp $31,300-310$.

Knobel, A., Heinz, A., Voss, M., 2008. Imaging the deluded brain. Eur Arch Psychiatry Clin Neurosci $258,76-80$.

Kunert, H.J., Norra, C., Hoff, P., 2007. Theories of Delusional Disorders. Psychopathology 40, 191202.

Ladavas, E., Zeloni, G., Farne, A., 1998. Visual peripersonal space centred on the face in humans. Brain 121, 2317-2323.

Lepping, P., Huber, M., Freudenmann, R.W., 2015. How to approach delusional infestation. Practical Pointer. BMJ Apr 1; 350, h1328.

Li, Y.O., Adali, T., Calhoun V.D., 2007. Estimating the number of independent components for functional magnetic resonance imaging data. Hum Brain Mapp 28, 1251-1266.

Mochizuki, H., Inui, K., Tanabe, H.C., Akiyama, L.F., Otsuru, N., Yamashiro, K., Sasaki, A., Nakata, H., Sadato, N., Kakigi, R., 2009. Time course of activity in itch-related brain regions: A combined MEG-fMRI study. J Neurophysiol 102, 2657-2666. 
Nagy, A., Eördegh, G., Paroczy, Z., Markus, Z., Benedek, G., 2006. Multisensory integration in the basal ganglia. Eur J Neurosci 24, 917-924.

Paulus, M.P., Stein, M.B., 2009. An insular view of anxiety. Biol Psychiatry 60, 383-387.

Pia, L., Garbarini, F., Burin, D., Fossataro, C., Berti, A., 2015. A predictive nature for tactile awareness? Insights from damaged and intact central-nervous-system functioning. Front Hum Neurosci 9, 287.

Preusser, S., Thiel, S.D., Rook, C., Roggenhofer, E., Kosatschek, A., Draganski, B., Blankenburg, F., Driver, J., Villringer, A., Pleger, B., 2015. The perception of touch and the ventral somatosensory pathway. Brain 138, 540-548.

Schmack, K., Gòmez-Carrillo de Castro, A., Rothkirch, M., Sekutowicz, M., Rössler, H., Haynes, J.D., Heinz, A., Petrovic, P., Sterzer, P., 2013. Delusions and the role of beliefs in perceptual inference. J Neurosci 33, 13701-13712.

Schneider, G., Stander, S., Burgmer, M., Driesch, G., Heuft, G., Weckesser M., 2008. Significant differences in central imaging of histamine-induced itch between atopic dermatitis and healthy subjects. Eur J Pain; 12, 834-841.

Skelton, M., Khokhar, W.A., Thacker, S.P., 2015. Treatments for delusional disorder. Cochrane Database Syst Rev 22, CD009785.

Spalletta, G., Piras, F., Alex Rubino, I., Caltagirone, C., Fagioli, S., 2013. Fronto-thalamic volumetry markers of somatic delusions and hallucinations in schizophrenia. Psychiatry Res 212, 54-64.

Su, K.P., Hsu, C.Y., Hsieh, S.C., Shen, W.W., 2001. Magnetic resonance imaging findings in patients with delusional disorders due to diffuse cerebrovascular diseases: a report of seven cases. Psychiatry Clin Neurosci 55, 121-126.

Trabert, W., 1995. 100 years of delusional parasitosis. Meta-analysis of 1.223 case reports. Psychopathology 28, 238-246.

Tzourio-Mazoyer, N., Landeau, B., Papathanassiou, D., Crivello, F., Etard, O., Delcroix, N., Mazojer, B., Joliot, M., 2002. Automated anatomical labeling of activations in SPM using a macroscopic anatomical parcellation of the MNI MRI single-subject brain. Neuroimage 15, 273-289. 
Vicens, V., Radua, J., Salvador, R., Anguera-Camos, M., Canales-Rodriguez, E.J., Sarro, S., Maristany, T., McKenna, P.J., Pomarol-Clotet, E., 2016. Structural and functional brain changes in delusional disorder. Br J Psych 208, 153-159.

Wicker, B., Keysers, C., Plailly, J., Royet, J.P., Gallese, V., Rizzolatti, G., 2003. Both of us disgusted in my insula: the common neural basis of seeing and feeling disgust. Neuron 40, 655-664.

Woods, S.W., 2003. Chlorpromazine equivalent doses for the newer atypicalantipsychotics, J Clin Psychiatry 64, 663-667.

Wolf, R.C., Huber, M., Depping, M.S., Thomann, P.A., Karner, M., Lepping, P., Freudenmann, R.W., 2013. Abnormal gray matter and white matter volume in delusional infestation. Prog Neuropsychopharmacol Biol Psychiatry 46, 19-24.

Wolf, R.C., Huber, M., Lepping, P., Sambataro, F., Depping, M.S., Karner, M., Freudenmann, R.W., 2014. Source-based morphometry reveals distinct patterns of aberrant brain volume in delusional infestation. Prog Neuropsychopharmacol Biol Psychiatry 48, 112-116.

XU, L., Groth, K.M., Pearlson, G., Schretlen, D.J., Calhoun, V.D., 2009. Source-based-morphometry: the use of independent component analysis to identify gray matter differences with application to schizophrenia. Hum Brain Mapp 30, 711-724.

Yoo, S.S., Freeman, D.K., McCarthy, J.J 3 rd ; Jolesz, F.A., 2003. Neural substrates of tactile imagery: a functional MRI study. Neuroreport 14, 581-585. 


\section{Figure legends}

Figure 1. Top: Regions showing lower gray matter volume (GMV) in patients with delusional infestation (DI) compared to healthy controls. Shown are results of $2^{\text {nd }}$ level between-group analyses, $\mathrm{p}<0.05$ corrected for spatial extent (see also Table 1 for detailed stereotaxic coordinates and Z-scores). The statistical maps are rendered onto the anatomical templates implemented in SPM8. The color bar represents T-values. Bottom: Regions showing lower gray matter volume (GMV) in patients with delusional disorders other than DI (NonDI-group) compared to healthy controls. Left: Results obtained across the entire patient sample. Right: Results obtained using a subset of patients $(n=13)$ presenting with prominent paranoid (persecutory) delusions. Shown are results of $2^{\text {nd }}$ level between-group analyses, $p<0.05$ corrected for spatial extent (see also Table 1 for detailed stereotaxic coordinates and Zscores). The statistical maps are rendered onto the anatomical templates implemented in SPM8. The color bar represents T-values.

Figure 2. Structural networks exhibiting differences in patients with delusional infestation (DI) compared to healthy controls and compared to patients with delusional disorders other than DI (NonDI-group) (see also Table 2 for detailed anatomical labels, coordinates and Zscores). Shown are the two GMV components which showed a significant group effect as a result of an ANOVA, $\mathrm{p}<0.05$, FDR-corrected.

* indicates significant between group differences as revealed by post-hoc Fisher's LSD tests. "n.s." indicates non-significant findings. For visualization purposes the components were thresholded at $Z>1.0$ and rendered onto the anatomical template implemented in GIFT. The color bar indicates the Z-value. L: left, R: right. 